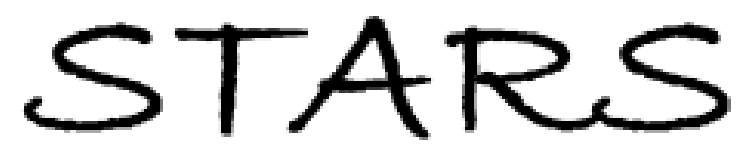

University of Central Florida

STARS

$1-1-2004$

\title{
Correlations between liquid crystal director reorientation and optical response time of a homeotropic cell
}

Haiying Wang

University of Central Florida

Thomas X. Wu

University of Central Florida

Xinyu Zhu

University of Central Florida

Shin-Tson Wu

University of Central Florida

Find similar works at: https://stars.library.ucf.edu/facultybib2000

University of Central Florida Libraries http://library.ucf.edu

This Article is brought to you for free and open access by the Faculty Bibliography at STARS. It has been accepted for inclusion in Faculty Bibliography 2000s by an authorized administrator of STARS. For more information, please contactSTARS@ucf.edu.

\section{Recommended Citation}

Wang, Haiying; Wu, Thomas X.; Zhu, Xinyu; and Wu, Shin-Tson, "Correlations between liquid crystal director reorientation and optical response time of a homeotropic cell" (2004). Faculty Bibliography 2000s. 4858.

https://stars.library.ucf.edu/facultybib2000/4858

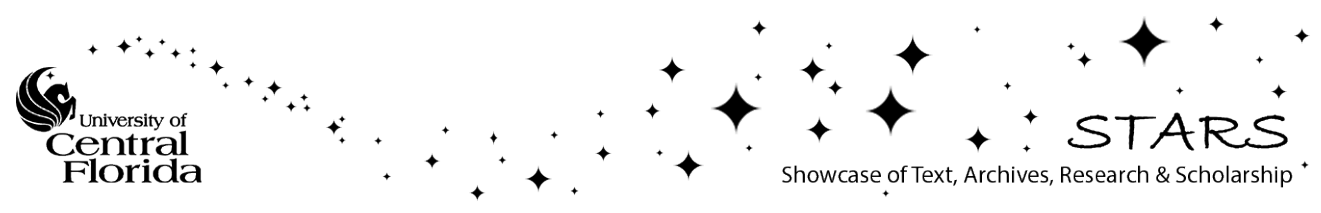




\section{Correlations between liquid crystal director reorientation and optical response time of a homeotropic cell}

Cite as: Journal of Applied Physics 95, 5502 (2004); https://doi.org/10.1063/1.1707210

Submitted: 25 August 2003. Accepted: 25 February 2004 . Published Online: 06 May 2004

Haiying Wang, Thomas X. Wu, Xinyu Zhu, and Shin-Tson Wu

\section{ARTICLES YOU MAY BE INTERESTED IN}

Anchoring energy and cell gap effects on liquid crystal response time

Journal of Applied Physics 101, 103110 (2007); https://doi.org/10.1063/1.2734870

Alignment layer effects on thin liquid crystal cells

Applied Physics Letters 92, 061102 (2008); https://doi.org/10.1063/1.2841642

Image sticking in liquid crystal displays with lateral electric fields

Journal of Applied Physics 116, 193102 (2014); https://doi.org/10.1063/1.4902083

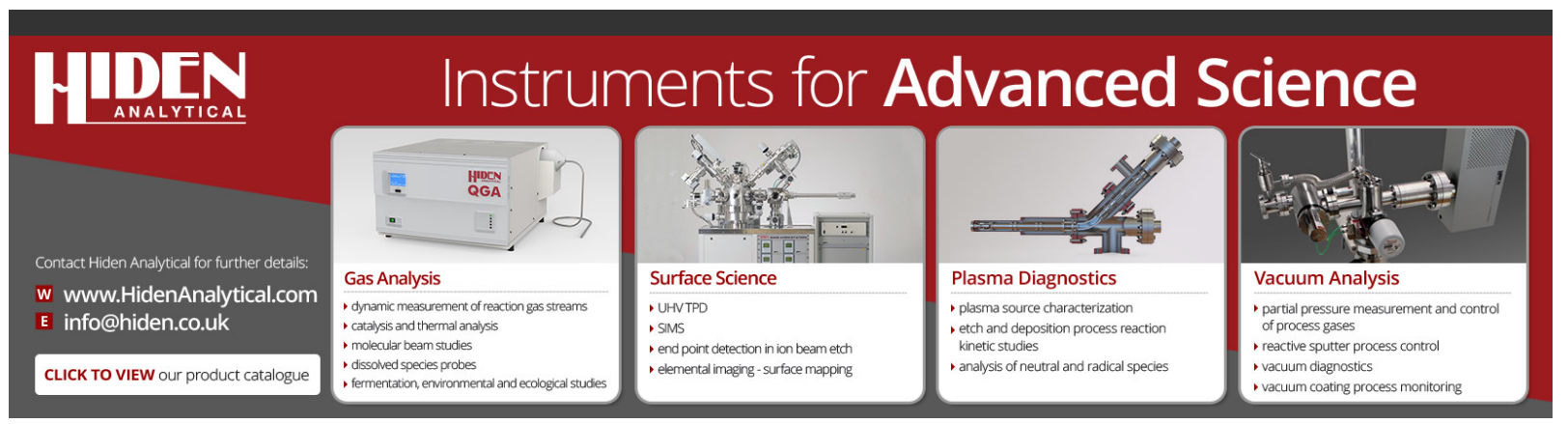




\title{
Correlations between liquid crystal director reorientation and optical response time of a homeotropic cell
}

\author{
Haiying Wang and Thomas X. Wu \\ School of Electrical Engineering and Computer Science, University of Central Florida, \\ Orlando, Florida 32816
}

Xinyu Zhu and Shin-Tson Wu $\mathrm{Wu}^{\mathrm{a}}$

School of Optics/CREOL, University of Central Florida, Orlando, Florida 32816

(Received 25 August 2003; accepted 25 February 2004)

\begin{abstract}
Correlations between the director reorientation time and its consequent optical response time (both decay and rise) of a homeotropic liquid crystal (LC) cell under crossed polarizers are derived theoretically based on small angle approximation. Results indicate that the optical response time is linearly proportional to the LC director reorientation time and is weakly dependent on the initial bias voltage. To validate the derived correlations, transient phase and transmittance responses at various bias voltages are analyzed numerically by solving the Erickson-Leslie equation. Pretilt angle is found to make an important contribution to the optical response time. Gray scale switching of the homeotropic cell is also investigated. (C) 2004 American Institute of Physics.
\end{abstract}

[DOI: $10.1063 / 1.1707210]$

\section{INTRODUCTION}

Response time is one of the most critical issues for nearly all the liquid crystal (LC) devices involving dynamic switching. Based on the small angle approximation, Jakeman and Raynes derived the LC director reorientation times. ${ }^{1}$ Since then, numerous papers dealing with LC response time have been published, however, the response time formula that most literatures refer to is the LC director reorientation time rather than the optical response time. For amplitude modulation, e.g., liquid crystal display devices, ${ }^{2}$ the LC device is usually sandwiched between two polarizers. The measured quantity is transmittance change and the associated dynamic response is optical rise or decay time. On the other hand, for a phase-only modulator such as optical phased arrays, ${ }^{3}$ the measured response time is phase change. There is no doubt that the optical response time for amplitude modulation and phase response time for phase modulation must be related to the LC director reorientation time. To quantify a display device, the rise and decay time is usually defined as intensity change between $10 \%$ and $90 \%$. However, the correlation between the director reorientation time and the optical and phase response time has not been carefully studied. Based on a simplified model, $\mathrm{Wu}^{4}$ found that the optical decay time could be $\sim 2 \times$ faster than the director reorientation time in a homogeneous LC cell. It is important to establish the detailed correlation between the LC director reorientation time and the optical and phase response time.

In this paper, we derived the analytical correlation between the director reorientation time and its consequent optical rise and decay times based on the small angle approximation. A vertical-aligned (VA, also known as homeotropic) ${ }^{5}$ nematic LC cell was used for these studies due to its simple electro-optic characteristics and widespread applications in

\footnotetext{
${ }^{a)}$ Electronic mail: swu@mail.ucf.edu
}

transmissive direct-view and reflective projection displays. ${ }^{6-8}$ To validate the derived correlations, in Sec. II we numerically solved the dynamic Erickson-Leslie equation. Results indicate that the optical response time is linearly proportional to the director reorientation time and is weakly dependent on the initial bias voltage. Pretilt angle effect is found to make an important contribution to the LC dynamics. Gray scale switching of the VA cell is also studied and results are discussed in Sec. III.

\section{THEORY}

When the backflow and inertial effects are ignored, the dynamics of the LC director reorientation is described by the following Erickson-Leslie equation: ${ }^{9,10}$

$$
\begin{aligned}
& \left(K_{11} \cos ^{2} \phi+K_{33} \sin ^{2} \phi\right) \frac{\partial^{2} \phi}{\partial z^{2}} \\
& +\left(K_{33}-K_{11}\right) \sin \phi \cos \phi\left(\frac{\partial \phi}{\partial z}\right)^{2} \\
& +\epsilon_{o} \Delta \epsilon E^{2} \sin \phi \cos \phi=\gamma_{1} \frac{\partial \phi}{\partial t},
\end{aligned}
$$

where $\gamma_{1}$ is the rotational viscosity, $K_{11}$ and $K_{33}$ represent the splay and bend elastic constants, respectively, $\epsilon_{o} \Delta \epsilon E^{2}$ is the electric field energy density, $\Delta \epsilon$ is the LC dielectric anisotropy, and $\phi$ is the tilt angle of the LC directors. In general, Eq. (1) can only be solved numerically. However, when the tilt angle is small $(\sin \phi \sim \phi)$ and $K_{33} \sim K_{11}$ (so-called small angle approximation), ${ }^{1}$ the Erickson-Leslie equation is reduced to

$$
K_{33} \frac{\partial^{2} \phi}{\partial z^{2}}+\epsilon_{o} \Delta \epsilon E^{2} \phi=\gamma_{1} \frac{\partial \phi}{\partial t} .
$$


Under such circumstances, both risetime and decay time have simple analytical solutions.

\section{A. Decay time}

When the electric field is switched off, i.e., $E=0$, Eq. (2) is further simplified as

$$
K_{33} \frac{\partial^{2} \phi}{\partial z^{2}}=\gamma_{1} \frac{\partial \phi}{\partial t} .
$$

The solution of Eq. (3) can be expressed as

$$
\phi(z, t) \cong \phi_{m} \sin \left(\frac{\pi z}{d}\right\rangle \exp \left(-\frac{t}{\tau_{o}}\right)
$$

with

$$
\tau_{o}=\frac{\gamma_{1} d^{2}}{K_{33} \pi^{2}}
$$

where $\phi_{m}$ is the maximum tilt angle of the LC directors in the response of the applied voltage, $d$ is the LC cell gap, $z$ is the position of the oriented LC layer under discussion, and $\tau_{o}$ is the LC director reorientation time $(1 \rightarrow 1 / e)$. It should be pointed out that in the Erickson-Leslie equation the strong surface anchoring and zero pretilt angle at the surface boundaries are assumed. Under such conditions, the Freedericksz transition threshold exists, ${ }^{11}$

$$
V_{\mathrm{th}}=\pi \sqrt{\frac{K_{33}}{\epsilon_{o} \Delta \epsilon}} .
$$

The time-dependent phase change associated with this angle change is described as follows:

$$
\Delta(t)=\frac{2 \pi}{\lambda} \int_{0}^{d}\left[\frac{n_{e} n_{o}}{\left(n_{o}^{2} \cos ^{2} \phi+n_{e}^{2} \sin ^{2} \phi\right)}-n_{o}\right] d z,
$$

where $n_{e}$ and $n_{o}$ are the refractive indices for the extraordinary and ordinary rays, respectively.

If a VA cell is initially biased at a voltage $\left(V_{b}\right)$ which is not too far above $V_{\text {th }}$, and the voltage is removed instantaneously at $t=0$, the transient phase change can be approximated from Eq. (7) as ${ }^{12}$

$$
\delta(t) \cong \delta_{o} \exp \left(-\frac{2 t}{\tau_{o}}\right)
$$

where $\delta_{o}$ is the net phase change from $V=V_{b}$ to $V=0$. From Eq. $(8)$, the phase decay time constant $(1 \rightarrow 1 / e)$ is $\tau_{o} / 2$ which is $2 \times$ faster than the LC reorientation time.

To find optical response time, we need to calculate the intensity change. The time-dependent normalized intensity change $I(t)$ of the VA cell under crossed polarizers can be calculated using the following relationship:

$$
I(t)=\sin ^{2}\left(\frac{\delta(t)}{2}\right)
$$

Substituting Eq. (8) into Eq. (9), we find

$$
I(t)=\sin ^{2}\left(\frac{\delta_{o} \exp \left(-\frac{2 t}{\tau_{o}}\right)}{2}\right) .
$$

In a display device, two definitions of response time are encountered: optical transmittance change from $90 \%$ to $10 \%$ or from $100 \%$ to $10 \%$. The process for correlating the optical response time to the director reorientation time is similar. Let us consider the former case first.

Based on Eq. (10), the normalized transmittance at $t$ $=0$ has the following simple expression:

$$
I_{o}=\sin ^{2}\left(\frac{\delta_{o}}{2}\right) .
$$

Let us assume from $t_{1}$ to $t_{2}$ the transmittance decays from $I_{1}=90 \%$ to $I_{2}=10 \%$. From Eq. (10), $I_{1}$ and $I_{2}$ have the following forms:

$$
\begin{aligned}
& I_{1}=0.9 I_{o}=\sin ^{2}\left(\frac{\delta_{o} \exp \left(-\frac{2 t_{1}}{\tau_{o}}\right)}{2}\right), \\
& I_{2}=0.1 I_{o}=\sin ^{2}\left(\frac{\delta_{o} \exp \left(-\frac{2 t_{2}}{\tau_{o}}\right)}{2}\right) .
\end{aligned}
$$

Using Eqs. (11), (12), and (13), we can easily solve the optical decay time $T_{\text {decay }}(90 \% \rightarrow 10 \%)$ as follows:

$$
T_{\text {decay }}=t_{2}-t_{1}=\frac{\tau_{o}}{2} \ln \left(\frac{\sin ^{-1}\left(\sqrt{0.9} \sin \left(\frac{\delta_{o}}{2}\right)\right)}{\sin ^{-1}\left(\sqrt{0.1} \sin \left(\frac{\delta_{o}}{2}\right)\right)}\right) .
$$

Equation (14) correlates the optical decay time to the LC director reorientation time $\left(\tau_{o}\right)$. Similarly, the optical decay time from $100 \%$ to $10 \%$ can be derived easily and result is shown below:

$$
T_{\text {decay }}=t_{1}=\frac{\tau_{o}}{2} \ln \left(\frac{\delta_{o} / 2}{\sin ^{-1}\left(\sqrt{0.1} \sin \left(\frac{\delta_{o}}{2}\right)\right)}\right) .
$$

From Eqs. (14) and (15), the optical decay time of a VA cell is linearly proportional to the director decay time. The initial phase retardation $\left(\delta_{o}\right)$ also plays an important role, but not too substantially. The detailed numerical results will be shown in Sec. III.

\section{B. Risetime}

Risetime is much more complicated to deal with than relaxation. The original small angle approximation used by Jakeman and Raynes for risetime is oversimplified. ${ }^{1}$ They have assumed that the LC director's tilt angle increases exponentially with time. This approximation is valid only in a very short time regime. Blinov has considered the second order term and the fact that the LC directors will eventually reach an equilibrium stage. Thus, Eq. (2) is rewritten as ${ }^{13}$ 


$$
\xi^{2} \frac{\partial^{2} \phi}{\partial z^{2}}+\phi-\frac{1}{2} \phi^{3}=\lambda \frac{\partial \phi}{\partial t},
$$

where

$$
\begin{gathered}
\lambda=\frac{\gamma_{1}}{\epsilon_{o} \Delta \epsilon E^{2}}, \\
\xi^{2}=\frac{K_{33}}{\epsilon_{o} \Delta \epsilon E^{2}} .
\end{gathered}
$$

In Eqs. (17) and (18), the electric field intensity $E$ is expressed as

$$
E=V / d,
$$

where the bias voltage $V$ should satisfy $\left(V-V_{\mathrm{th}}\right) / V_{\mathrm{th}} \leqslant 1$. Under such a circumstance, the solution of Eq. (16) can be approximated as

$$
\phi=\phi_{m}(t) \sin \left(\frac{\pi z}{d}\right) .
$$

Substituting Eq. (20) to Eq. (16), we obtain

$$
\left[1-\left(\frac{V_{\mathrm{th}}}{V}\right)^{2}\right] \phi_{m}-\frac{\phi_{m}^{3}}{2}=\lambda \frac{d \phi_{m}}{d t} .
$$

Equation (21) has following solution:

$$
\phi_{m}^{2}=\frac{\phi_{\infty}^{2}}{1+\left[\frac{\phi_{\infty}^{2}}{\phi_{o}^{2}}-1\right] \exp \left(-\frac{2 t}{\tau_{r}}\right)},
$$

where $\phi_{\infty}=\phi(t \rightarrow \infty)$ is the steady-state value of $\phi_{m}$ corresponding to the biased voltage, $\phi_{o}=\phi(t=0)$ is the initial directors fluctuation, and $\tau_{r}$ is the directors risetime,

$$
\tau_{r}=\frac{\gamma_{1}}{\left|\epsilon_{o}\right| \Delta \epsilon\left|E^{2}-\frac{\pi^{2}}{d^{2}} K\right|}=\frac{\tau_{o}}{\left|\left(\frac{V}{V_{\mathrm{th}}}\right)^{2}-1\right|} .
$$

Under small angle approximation, the transient phase change is obtained as

$$
\delta(t) \cong \frac{\delta_{o}}{1+\left[\frac{\phi_{\infty}^{2}}{\phi_{o}^{2}}-1\right] \exp \left(-\frac{2 t}{\tau_{r}}\right)},
$$

where $\delta_{o}$ is the total phase change from the voltage-off state to the voltage-on state. By substituting Eq. (24) into Eq. (8), we obtain the transient transmittance state

$$
I(t)=\sin ^{2}\left(\frac{\delta_{o} / 2}{1+\left[\frac{\phi_{\infty}^{2}}{\phi_{o}^{2}}-1\right] \exp \left(-\frac{2 t}{\tau_{r}}\right)}\right) .
$$

At $t \rightarrow \infty$, the exponential term in Eq. (25) vanishes and $I(t)$ reaches a plateau

$$
I(\infty)=\sin ^{2}\left(\frac{\delta_{o}}{2}\right) .
$$

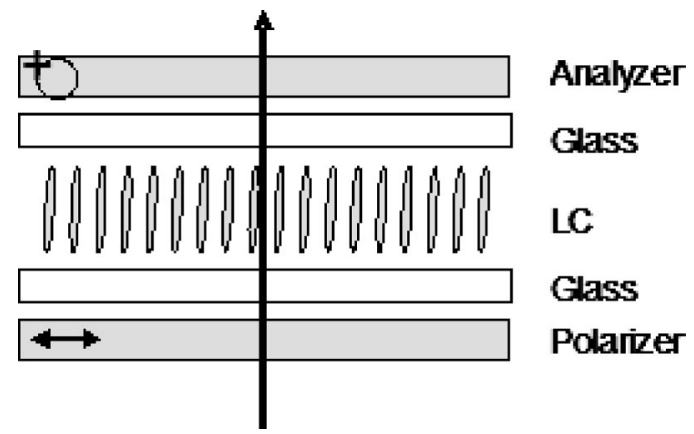

FIG. 1. The VA cell used for this study. The LC cell is sandwiched between crossed polarizers. The inner side of each glass substrate is coated with a thin layer of indium-tin-oxide and polyimide for producing homeotropic alignment. The LC has a small pretilt angle.

To solve the optical risetime, let us assume the transmittance rises from $I_{1}$ to $I_{2}$ as the time increases from $t_{1}$ to $t_{2}$. Substituting $t_{1}$ and $t_{2}$ to Eq. (25), we obtain the corresponding transmittance at $10 \%$ and $90 \%$ :

$$
\begin{aligned}
& I_{1}=0.1 I_{o}=\sin ^{2}\left(\frac{\delta_{o} / 2}{1+\left[\frac{\phi_{\infty}^{2}}{\phi_{o}^{2}}-1\right] \exp \left(-\frac{2 t_{1}}{\tau_{r}}\right)}\right), \\
& I_{2}=0.9 I_{o}=\sin ^{2}\left(\frac{\delta_{o} / 2}{1+\left[\frac{\phi_{\infty}^{2}}{\phi_{o}^{2}}-1\right] \exp \left(-\frac{2 t_{2}}{\tau_{r}}\right)}\right) .
\end{aligned}
$$

By solving $t_{1}$ and $t_{2}$ from Eqs. (27) and (28), we derive the optical risetime $T_{\text {rise }}(10 \% \rightarrow 90 \%)$ as

$$
\begin{aligned}
T_{\text {rise }} & =t_{2}-t_{1} \\
& =\frac{1}{2} \frac{\tau_{o}}{\left|\left(\frac{V}{V_{\mathrm{th}}}\right)^{2}-1\right|} \ln \left(\frac{\frac{\delta_{o} / 2}{\sin ^{-1}\left(\sqrt{0.1} \sin \left(\frac{\delta_{o}}{2}\right)\right)}-1}{\frac{\delta_{o} / 2}{\sin ^{-1}\left(\sqrt{0.9} \sin \left(\frac{\delta_{o}}{2}\right)\right)}-1}\right) .
\end{aligned}
$$

Equation (29) correlates the optical risetime $\left(T_{\text {rise }}\right)$ to the commonly used director risetime $\left(\tau_{r}\right)$ as described in Eq. (23). Basically, it is a linear relationship except for the additional logarithm term of the phase dependence. As will be discussed in Sec. III, this phase dependence is relatively modest.

\section{RESULTS AND DISCUSSIONS}

To validate Eqs. (14) and (29), we numerically solve Eq. (1) using the finite element method (FEM). ${ }^{14}$ Once the LC director distribution is obtained, we then use the extended Jones matrix method ${ }^{15,16}$ to calculate the transient phase change $\delta(t)$. Figure 1 shows the system configuration of the VA LC under study. A commercial Merck negative nematic MLC-6608 mixture was used in our computer simulations. The material parameters of MLC 6608 are: $n_{o}=1.4748, n_{e}$ 


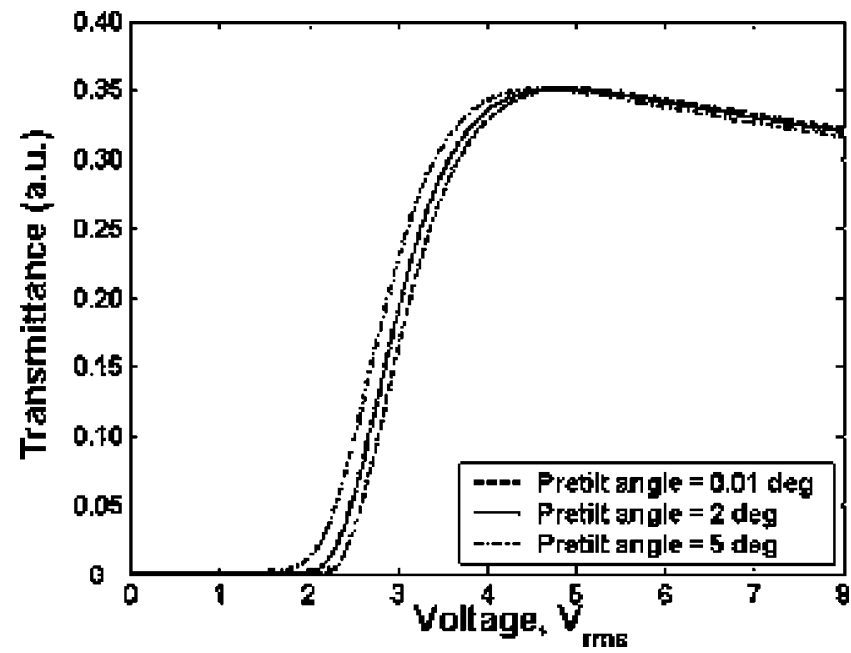

FIG. 2. The simulated voltage-dependent transmittance of a VA cell at $\lambda=550 \mathrm{~nm}$ with three different pretilt angles, $\alpha=0.01^{\circ}$ (dashed line), $2^{\circ}$ (solid line), and $5^{\circ}$ (dashed-dotted line). The parameters used for simulations are listed.

$=1.5578$, the dielectric anisotropy $\Delta \epsilon=-4.2$, the rotational viscosity $\gamma_{1}=186 \mathrm{mPas}$ at $20^{\circ} \mathrm{C}$, the splay elastic constant $k_{11}=16.7 \times 10^{-12} \mathrm{~N}$, twist elastic constant $k_{22}=7.0$ $\times 10^{-12} \mathrm{~N}$, and bend elastic constant $k_{33}=18.1 \times 10^{-12} \mathrm{~N}$. The buffing induced pretilt angle is assumed to be $2^{\circ}$ from surface normal unless otherwise mentioned.

For a thin-film-transistor liquid crystal display using transmissive VA cell, the on-state voltage is preferred to be restricted to $\sim 5 V_{\text {rms }}$ for the interest of low power consumption. Therefore, we choose $d \Delta n=0.7 \lambda$, which is slightly larger than the required half-wave phase retardation in order to reduce the on-state voltage. By using MLC-6608, the corresponding cell gap is $d=4.64 \mu \mathrm{m}$ and the total phase retardation is $\delta=1.4 \pi$ at $\lambda=550 \mathrm{~nm}$. Based on Eq. (15), the threshold voltage $V_{\text {th }}$ is calculated to be $2.19 V_{\text {rms }}$. At the first transmission peak (i.e., $\delta=\pi$ ), $V=2.146 V_{\text {th }}$. We have also studied the response time between gray scales.

\section{A. Pretilt angle effect}

For a VA cell, pretilt angle $(\alpha)$ affects the device contrast ratio and response time. Here, we define pretilt angle as the angle of the LC directors deviated from cell normal. If $\alpha=0$, it implies that the LC directors are aligned perpendicular to the substrate surfaces. Figure 2 plots the voltage-dependent transmittance (or called VT curve) at $\alpha=0.01^{\circ}, 2^{\circ}$, and $5^{\circ}$. Please note that $\tau_{o}$ was derived by assuming $\alpha=0$. However, in a real LC device a small pretilt angle is required for LC directors to relax back without creating domains. Therefore, we use $\alpha=0.01^{\circ}$ to animate the results for $\alpha=0$. As the pretilt angle deviates from the cell normal, the threshold behavior is gradually smeared and the turn-on voltage is decreased. We have calculated the quantitative LC director reorientation time and optical response time at various voltages for different pretilt angles. However, it will be tedious to tabulate all the results here. To find the tendency while not losing generality, we choose the simulation results with $\alpha=0.01^{\circ}$ and $2^{\circ}$, as shown in Tables I and II, respectively. From Table I, the phase decay time data (the $t_{p} / \tau_{o}$ column)
TABLE I. Simulation results of phase decay time and optical decay time at different voltages of a VA cell. LC: MLC-6608, $d=4.64 \mu \mathrm{m}$, pretilt angle $\alpha=0.01^{\circ}$ and $V_{\mathrm{th}}=2.19 V_{\mathrm{rms}}$. Here, $t_{p}$ is the phase decay time, $T_{\text {decay }}$ is the optical decay time, and $\tau_{o}=22.4 \mathrm{~ms}$ is the director's decay time as defined in Eq. (5).

\begin{tabular}{ccccccc}
\hline \hline $\begin{array}{c}\text { Voltage } \\
\left(V_{\text {rms }}\right)\end{array}$ & $V / V_{\text {th }}$ & $\begin{array}{c}\text { Phase } \\
(\pi)\end{array}$ & $\begin{array}{c}t_{p}\left(1 \rightarrow 1 / e^{2}\right) \\
(\mathrm{ms})\end{array}$ & $t_{p} / \tau_{o}$ & $\begin{array}{c}T_{\text {decay }}(90 \rightarrow 10 \%) \\
(\mathrm{ms})\end{array}$ & $T_{\text {decay }} / \tau_{o}$ \\
\hline 2.30 & 1.05 & 0.0640 & 22.6 & 1.01 & 15.1 & 0.67 \\
2.40 & 1.10 & 0.1239 & 22.8 & 1.02 & 14.2 & 0.63 \\
2.50 & 1.14 & 0.1846 & 23.0 & 1.03 & 14.0 & 0.62 \\
2.60 & 1.19 & 0.2452 & 23.3 & 1.04 & 14.0 & 0.62 \\
2.80 & 1.28 & 0.3625 & 23.7 & 1.06 & 14.5 & 0.65 \\
2.90 & 1.32 & 0.4181 & 24.0 & 1.07 & 14.7 & 0.66 \\
3.00 & 1.37 & 0.4709 & 24.3 & 1.08 & 15.1 & 0.67 \\
3.20 & 1.46 & 0.5675 & 24.8 & 1.11 & 15.9 & 0.71 \\
3.50 & 1.60 & 0.6892 & 25.5 & 1.14 & 17.0 & 0.76 \\
3.70 & 1.69 & 0.7558 & 25.9 & 1.16 & 17.7 & 0.79 \\
4.00 & 1.83 & 0.8375 & 26.5 & 1.18 & 18.4 & 0.82 \\
4.40 & 2.01 & 0.9199 & 27.2 & 1.21 & 19.2 & 0.86 \\
4.70 & 2.15 & 0.9672 & 27.6 & 1.23 & 19.5 & 0.87 \\
\hline \hline
\end{tabular}

agree with the LC director decay time quite well in the low voltage regime. Here, to compare with $\tau_{o}$, we simply calculated the phase decay from 1 to $1 / e^{2}$ [Eq. (8)]. As the voltage increases, the $t_{p} / \tau_{o}$ ratio gradually deviates from unity. At $V / V_{\text {th }} \sim 1.6, t_{p} / \tau_{o}$ increases by $\sim 14 \%$. At $\delta \sim \pi$, the phase decay time is $\sim 23 \%$ longer than $\tau_{o}$. On the other hand, the optical decay time (from $90 \%$ to $10 \%$ ) remains relatively constant $\left(T_{\text {decay }} / \tau_{o} \sim 0.65 \pm 0.03\right)$ in the $V_{\text {th }}<V<1.4 V_{\text {th }}$ regime. As the voltage increases to $V / V_{\text {th }} \sim 2.15, T_{\text {decay }} / \tau_{o}$ increases to 0.87 .

Table II shows the calculated results for $\alpha=2^{\circ}$. A similar trend as that of $\alpha=0.01^{\circ}$ is still observed except that both phase and optical decay times are somewhat slower. The theoretical director decay time $\tau_{o}$ is assumed unchanged. The slower phase and optical decay time is believed to originate from the slightly weaker restoring elastic torque due to the increased pretilt angle.

Figures 3(a) and 3(b) plot the calculated optical decay time $(90 \% \rightarrow 10 \%)$ and risetime $(10 \rightarrow 90 \%)$, respectively, as a function of $V / V_{\text {th }}$ at $\alpha=1^{\circ}, 2^{\circ}, 3^{\circ}$, and $5^{\circ}$ pretilt angles. The Erickson-Leslie equation was used for these calculations. In general, at a given $V / V_{\text {th }}$ a smaller pretilt angle would lead to

TABLE II. Same as Table I except the pretilt angle $\alpha=2^{\circ}$.

\begin{tabular}{ccccccc}
\hline \hline $\begin{array}{c}\text { Voltage } \\
\left(V_{\text {rms }}\right)\end{array}$ & $V / V_{\text {th }}$ & $\begin{array}{c}\text { Phase } \\
(\pi)\end{array}$ & $\begin{array}{c}t_{p}\left(1 \rightarrow 1 / e^{2}\right) \\
(\mathrm{ms})\end{array}$ & $t_{p} / \tau_{o}$ & $\begin{array}{c}T_{\text {decay }}(90 \rightarrow 10 \%) \\
(\mathrm{ms})\end{array}$ & $T_{\text {decay }} / \tau_{o}$ \\
\hline 2.30 & 1.05 & 0.1425 & 26.4 & 1.18 & 16.1 & 0.72 \\
2.40 & 1.10 & 0.1958 & 26.1 & 1.17 & 15.7 & 0.70 \\
2.50 & 1.14 & 0.2516 & 26.0 & 1.16 & 15.5 & 0.69 \\
2.60 & 1.19 & 0.3081 & 26.0 & 1.16 & 15.6 & 0.70 \\
2.80 & 1.28 & 0.4184 & 26.1 & 1.17 & 15.9 & 0.71 \\
2.90 & 1.32 & 0.4706 & 26.3 & 1.17 & 16.3 & 0.73 \\
3.00 & 1.37 & 0.5202 & 26.4 & 1.18 & 16.5 & 0.74 \\
3.20 & 1.46 & 0.6108 & 26.8 & 1.20 & 17.3 & 0.77 \\
3.50 & 1.60 & 0.7246 & 27.3 & 1.22 & 18.4 & 0.82 \\
3.70 & 1.69 & 0.7869 & 27.7 & 1.24 & 19.0 & 0.85 \\
4.00 & 1.83 & 0.8632 & 28.2 & 1.26 & 19.8 & 0.88 \\
4.40 & 2.01 & 0.9404 & 28.9 & 1.29 & 20.3 & 0.91 \\
4.70 & 2.15 & 0.9849 & 29.3 & 1.31 & 20.5 & 0.92 \\
\hline \hline
\end{tabular}



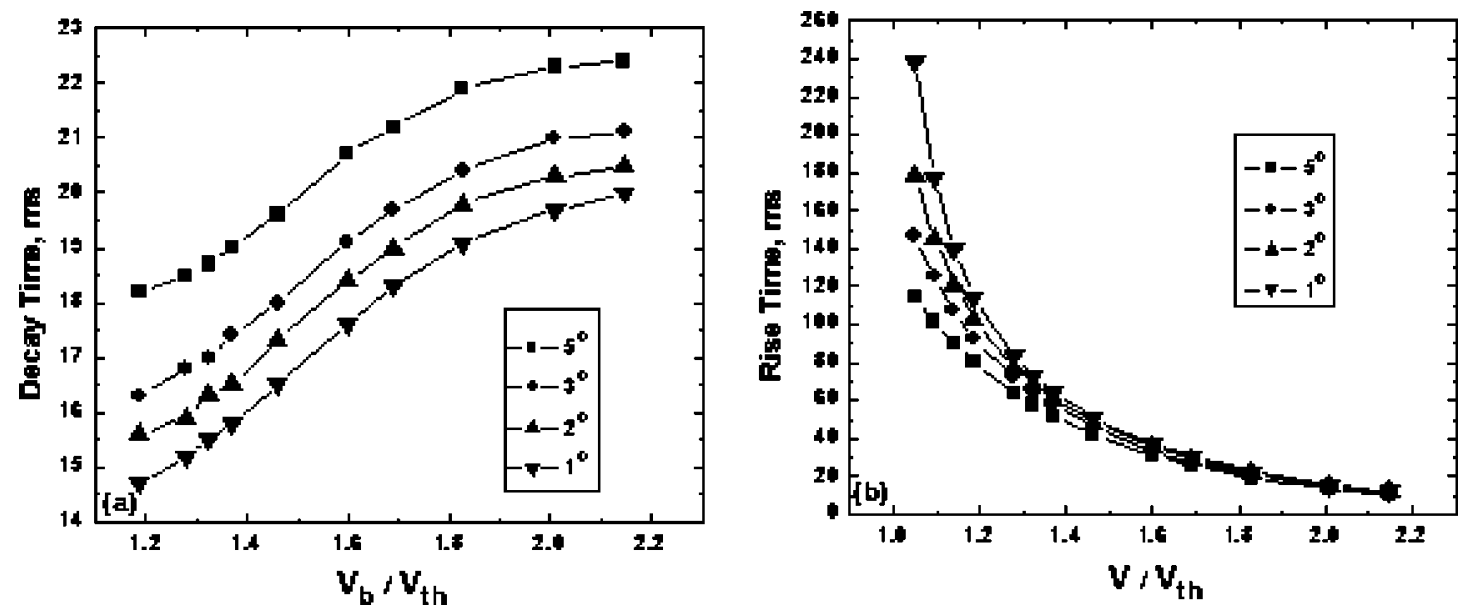

FIG. 3. (a) Optical decay time $(90 \% \rightarrow 10 \%)$ and (b) risetime $(10 \% \rightarrow 90 \%)$ as a function of $V / V_{\text {th }}$ at four different pretilt angles, $\alpha=1^{\circ}, 2^{\circ}, 3^{\circ}$, and $5^{\circ}$.

a faster decay time but slower risetime. In the vicinity of threshold, the risetime is particularly slow, as described in Eq. (23). As the voltage increases, the optical risetime is decreased rapidly. At $V \sim 2.2 V_{\text {th }}$ (peak transmittance), the risetime is reduced to $\sim 10 \mathrm{~ms}$.

Strictly speaking, the threshold behavior exists only when the pretilt angle is zero. However, in most LC devices a nonzero pretilt angle is required in order to avoid domain formation during molecular reorientation. The free relaxation time $\tau_{o}$ is derived based on the assumptions that $\alpha=0$ and the applied voltage is not too far above the threshold. In reality, these assumptions may not be valid. Taking into account the pretilt angle effect, we modify the free relax time according to the following equation:

$$
\tau_{o}^{*}=\beta \tau_{o},
$$

where $\tau_{o}$ is the free relaxation time when pretilt angle is zero, and can be calculated according to Eq. (5). In Eq. (30), $\beta$ is dependent on the pretilt angle. Since most of display cells have a pretilt angle, this correction factor is necessary to match theory with experimental results. ${ }^{17}$ We have used the Erickson-Leslie equation to calculate the LC response time including pretilt angle effect but without using the small angle approximation. The $\beta$ values we found are listed in Table III. At a very small pretilt angle $\alpha \sim 0.01^{\circ}, \tau_{o}^{*} \sim \tau_{o}$; i.e., the correction factor $\beta=1$, as expected. As the pretilt angle increases, $\beta$ gradually increases. At $\alpha=5^{\circ}, \beta$ is found to be higher than the ideal value, which is unity, by nearly $30 \%$.

The pretilt angle is dependent on the polyimide alignment layer, rubbing strength, and LC material employed. ${ }^{18,19}$ For a given polyimide alignment layer, different LC materi-

TABLE III. Pretilt angle effect on the LC director's decay time $\tau_{o}^{*}$.

\begin{tabular}{cl}
\hline \hline Pretilt angle $\alpha\left(^{\circ}\right)$ & $\tau_{o}^{*} / \tau_{o}$ \\
\hline 0.01 & 1 \\
1 & 1.10 \\
2 & 1.16 \\
3 & 1.21 \\
5 & 1.30 \\
\hline
\end{tabular}

als may have a slightly different pretilt angle depending on the molecular interactions. The typical pretilt angle for a VA cell is $\sim 2^{\circ}$. Thus, $\beta \sim 1.16$ has been taking into consideration whenever we calculated the response time of a VA cell with $\alpha=2^{\circ}$.

\section{B. Gray scale switching}

The beauty of a nematic LCD is that it has natural gray scales. Each primary color (red, green, and blue) can display 8-bits gray scales. Thus, a full-color display with 16 million colors can be obtained. To investigate gray scale switching, we divide the voltage-dependent transmittance curve into eight equal intensity gray levels, as shown in Fig. 4. Level 1 represents the dark state and level 8 for the brightest state. The maximum transmission shown in Fig. 4 is 35\% after taking the absorption of the polarizer and analyzer into consideration.

Table IV lists the calculated optical response time using the finite element and finite difference time domain methods for both decay and rise processes of the eight-level gray scales. The data in the right top triangle represent the rise time, while the left bottom are the decay time. The risetime from gray level 1 to 2 is the slowest because the applied

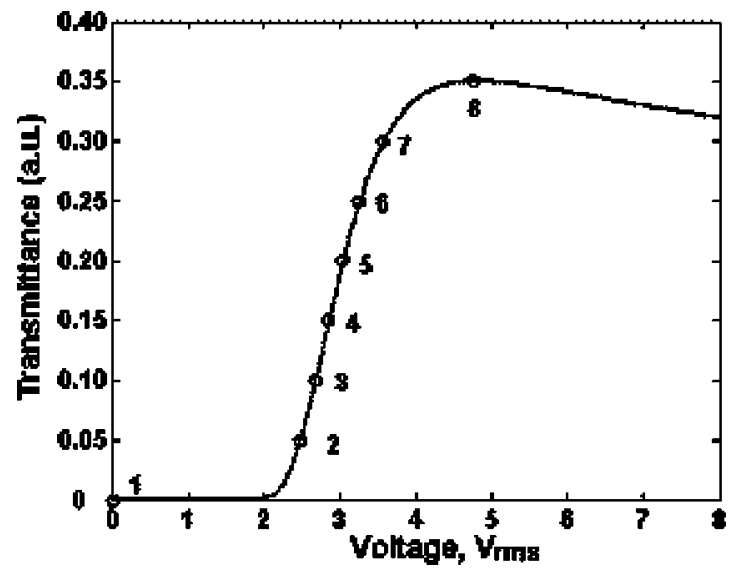

FIG. 4. The eight gray levels of the VA cell at $\lambda=550 \mathrm{~nm}$ LC: MLC-6608, $d=4.64 \mu \mathrm{m}$ and pretilt angle $\alpha=2^{\circ}$. 
TABLE IV. The calculated eight gray level optical risetime $(10 \% \rightarrow 90 \%)$ and decay time $(90 \% \rightarrow 10 \%)$ of the VA cell shown in Fig. 4.

\begin{tabular}{|c|c|c|c|c|c|c|c|c|c|}
\hline & & \multicolumn{8}{|c|}{ Risetime, ms } \\
\hline & & 1 & 2 & 3 & 4 & 5 & 6 & 7 & 8 \\
\hline \multirow[t]{8}{*}{ Decay time, $\mathrm{ms}$} & 1 & & 124.5 & 90.7 & 72.1 & 58 & 45.7 & 33.1 & 11.3 \\
\hline & 2 & 15.5 & & 69.4 & 58.9 & 49.2 & 39.8 & 29.2 & 9.6 \\
\hline & 3 & 15.7 & 70.8 & & 53.6 & 45.5 & 37.1 & 27.5 & 8.6 \\
\hline & 4 & 16.1 & 64.4 & 57.5 & & 43.4 & 35.7 & 26.6 & 8 \\
\hline & 5 & 16.7 & 60.2 & 54.7 & 48.5 & & 34.9 & 26.2 & 7.4 \\
\hline & 6 & 17.5 & 57.4 & 52.9 & 47.4 & 41.3 & & 26.2 & 7.0 \\
\hline & 7 & 18.6 & 55.8 & 52.1 & 47.1 & 41.6 & 35.2 & & 6.4 \\
\hline & 8 & 20.5 & 55.9 & 53 & 48.7 & 43.8 & 38.3 & 31.3 & \\
\hline
\end{tabular}

voltage is so close to the threshold voltage. In a VA cell, gray scale $1 \rightarrow 2$ represents the switching from the darkest state to the second darkest state. Although it is slow, it is forgiven because human eye could not resolve this change too well. In the high voltage regime, gray scale switching is relatively fast. The actual switching time depends on the cell gap and viscoelastic coefficient $\left(\gamma_{1} / K_{33}\right)$ of the LC material employed. To improve the switching speed between gray levels, the overdrive and undershoot method has been proposed and implemented in real display devices. ${ }^{20,21}$ The data in the first row and the first column will be further used in our correlation.

\section{Detailed correlations}

In this section, we show the detailed simulation results between the director reorientation time and optical response time. The Erickson-Leslie equation was used for these calculations. A good correlation between the LC director reorientation time and optical response time is found.

\section{Decay time}

In the small angle approximation, one of the important assumptions is $\sin (\theta) \sim \theta$. Under such a circumstance, the analytical form of LC director reorientation time constant $\tau_{o}$ can be derived. However, the LC director reorientation time is difficult to measure directly in an experiment. For display applications, the optical response time is a more practical term. How to correlate the LC director reorientation time to the measurable optical response time is an important task.

Figure 5(a) depicts the director distribution $(\phi(z))$ as a function of normalized cell gap $(z / d)$ at $V \sim 1.37 V_{\text {th }}$. Although the voltage is not too high from threshold, a large director deformation has already occurred. In the middle layer, the maximum director tilt angle $\left(\phi_{m}\right)$ has reached $\sim 53^{\circ}$. Therefore, it is difficult to foresee whether the small angle approximation still holds. If it does, then Eq. (8) should be valid and $\ln \left[\delta_{o} / \delta(t)\right]$ should be a linear function of time with slope equal to $2 / \tau_{o}$. From the slope measurement, $\tau_{o}$ can be extracted.

In experiment, the VA cell sandwiched between crossed polarizers is biased at a voltage $V_{b}$. When the LC cell relaxes from $V_{b}$ to 0 , the total phase change is $\delta_{o}$. For a VA cell intended for intensity modulation, $\delta_{o} \leqslant \pi$. When the voltage is released instantaneously at $t=0$, the timedependent transmittance is recorded. This time-dependent transmittance can be converted to the transient phase decay described by $\delta(t)$. Figure 5(b) plots the calculated $\ln \left[\delta_{o} / \delta(t)\right]$ as a function of time for the VA cell. Indeed, a straight line with slope of $0.0755 / \mathrm{ms}$ is obtained. Based on this slope, $\tau_{o}=26.5 \mathrm{~ms}$ is found. Using the LC parameters, we find $\tau_{o}$ $=22.4 \mathrm{~ms}$ from Eq. (5) and $\tau_{o}^{*}=26 \mathrm{~ms}$ from Eq. (28) with $\beta=1.16$ because of the $2^{\circ}$ pretilt angle. The agreement between the small angle approximation and the EricksonLeslie equation is amazingly good in this case.

Next, we validate the correlation between the optical decay time $\left(T_{\text {decay }}\right)$ and the director's decay time $\left(\tau_{o}\right)$, as expressed in Eq. (14). If we neglect the logarithm term, then $T_{\text {decay }}=0.5 \tau_{o}$; the observed optical response time is $2 \times$ shorter than that of the LC director decay time. With the phase-dependent term included, the change is still not too significant. Figure 6 plots $T_{\text {decay }} / \tau_{o}$ at different $\delta_{o}$, as described in Eq. (14). In Fig. 6, circles represent the simulation results using the Erickson-Leslie equation, while the solid line represents the small angle approximation. In the small
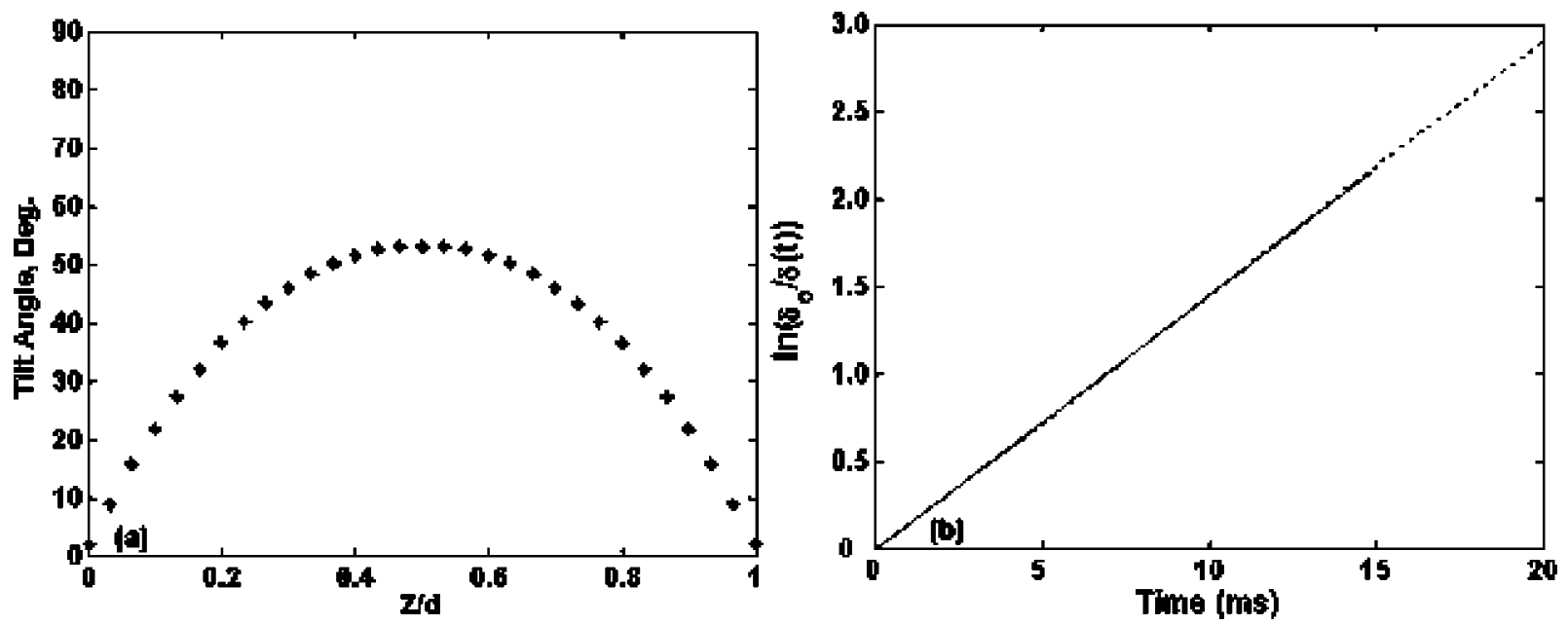

FIG. 5. (a) The calculated LC director distribution $\phi(z)$ as a function of normalized cell gap $(z / d)$. (b) Time-dependent $\ln \left[\delta_{o} / \delta(t)\right]$ of the VA cell. Dots are calculated data and solid line is the fitting curve. From the slope of the straight line, $\tau_{o}^{*}$ is found to be $\sim 26 \mathrm{~ms}$. 


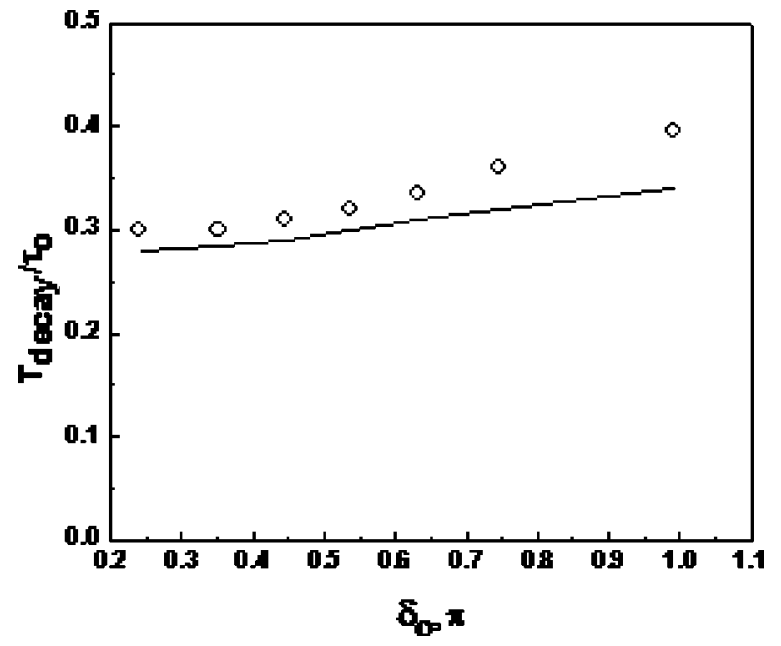

FIG. 6. The correlation of the optical decay time $T_{\text {decay }}(90 \% \rightarrow 10 \%)$ vs the LC director reorientation time $\left(\tau_{o}\right)$ as a function of $\delta_{o}$. Circles represent the simulation results using the Erickson-Leslie equation, while the solid line is the correlation obtained from the small angle approximation [Eq. (14)].

$\delta_{o}$ region, i.e., $V$ is not too far above $V_{\text {th }}$, the agreement between these two methods is reasonably good. As $\delta_{o}$ increases, the discrepancy increases slightly. At the biased voltage $V_{b} / V_{\text {th }}=2.146$ which corresponds to $\delta_{o} \sim 1 \pi$, the maximum error observed is $\sim 14 \%$.

\section{Risetime}

Risetime is much more difficult to solve than the decay time because it also depends on the applied voltage. Equation (29) correlates the optical risetime with the LC director risetime. At a given voltage, the optical risetime is $2 \times$ shorter than the LC director risetime if we neglect the logarithm term. Even the phase dependence term is included, the results are not affected too greatly. Figure 7 depicts the ratio of $T_{\text {rise }} / \tau_{o}$ at different $\delta_{o}$ as described by Eq. (29). In Fig. 7, circles represent the simulation results using the EricksonLeslie equation, while the solid line represents the small angle approximation. A very good agreement is obtained except in the near threshold region. When $V_{b}$ gets close to $V_{\text {th }}$, the pretilt angle effect becomes more pronounced. In our simulations, pretilt angle is assumed to be $2^{\circ}$. Due to the smeared and decreased threshold, the required voltage for obtaining $\delta_{o} \sim 0.25 \pi$ is lower. As a result, the calculated risetime is longer than $\tau_{o}$, as indicated by Eq. (23).

\section{CONCLUSION}

We have derived the correlations between the LC director reorientation time and the optical response (both decay and rise) time of a vertically aligned cell. Results indicate that the optical response time ( $T_{\text {rise }}$ and $\left.T_{\text {decay }}\right)$ is linearly proportional to the $\mathrm{LC}$ director reorientation time. The initial bias voltage effect is not too strong. Pretilt angle is found to

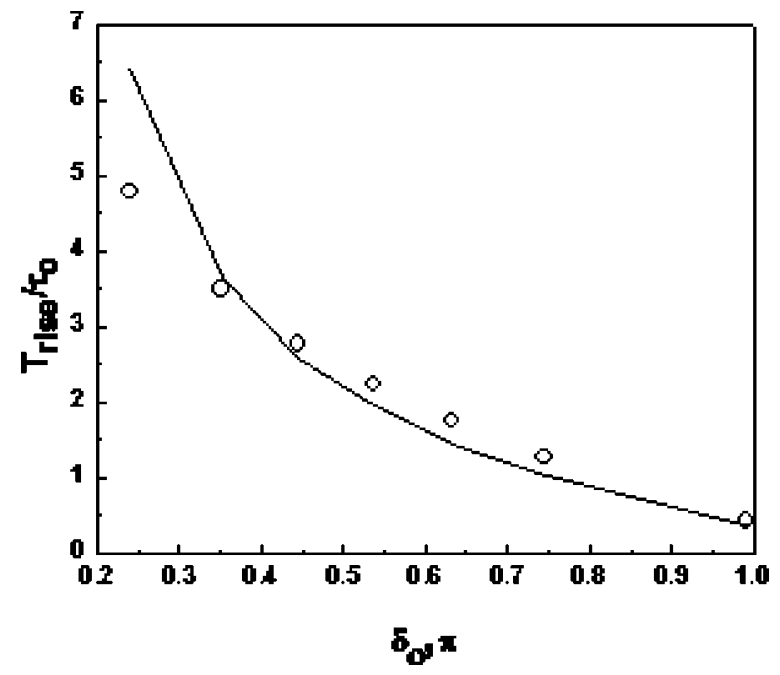

FIG. 7. The correlation of optical risetime $T_{\text {rise }}(10 \% \rightarrow 90 \%)$ vs the director reorientation time $\left(\tau_{o}\right)$ as a function $\delta_{o}$. Circles represent the simulation results using the Erickson-Leslie equation, while the solid line is the correlation obtained from the small angle approximation [Eq. (29)].

make an important impact to the LC dynamics. To correct for the pretilt angle effect, a modified rotational viscosity needs to be used.

\section{ACKNOWLEDGMENT}

The authors are indebted to the AFOSR for financial support under Contract No. F49620-01-1-0377.

${ }^{1}$ E. Jakeman and E. P. Raynes, Phys. Lett. 39A, 69 (1972).

${ }^{2}$ S. T. Wu and D. K. Yang, Reflective Liquid Crystal Displays (Wiley, New York, 2001).

${ }^{3}$ P. F. McManamon, T. A. Dorschner, D. L. Corkum, L. Friedman, D. S. Hobbs, M. Holz, S. Liberman, H. Q. Nguyen, D. P. Resler, R. C. Sharp, and E. A. Watson, Proc. IEEE 84, 268 (1996).

${ }^{4}$ S. T. Wu, J. Appl. Phys. 60, 1836 (1986).

${ }^{5}$ M. F. Schiekel and K. Fahrenschon, Appl. Phys. Lett. 19, 391 (1971).

${ }^{6}$ A. Takeda, S. Kataoka, T. Sasaki, H. Chida, H. Tsuda, K. Ohmuro, Y. Koike, T. Sasabayashi, and K. Okamoto, Soc. Inform. Display Technical Digest 29, 1077 (1998).

${ }^{7}$ J. O. Kwag, K. C. Shin, J. S. Kim, S. G. Kim, and S. S. Kim, Soc. Inform. Display Technical Digest 31, 256 (2000).

${ }^{8}$ R. D. Sterling and W. P. Bleha, Soc. Inform. Display Technical Digest 31, 310 (2000).

${ }^{9}$ J. L. Erickson, Trans. Soc. Rheol. 5, 23 (1961).

${ }^{10}$ F. M. Leslie, Arch. Ration. Mech. Anal. 28, 265 (1968).

${ }^{11}$ V. Freedericksz and V. Zolina, Trans. Faraday Soc. 29, 919 (1933).

${ }^{12}$ S. T. Wu and C. S. Wu, Phys. Rev. A 42, 2219 (1990).

${ }^{13}$ L. M. Blinov, Electro-Optical and Magneto-Optical Properties of Liquid Crystals (Wiley, New York, 1983).

${ }^{14}$ J. Jing, The Finite Element Method in Electromagnetics (Wiley, New York, 1993).

${ }^{15}$ R. C. Jones, J. Opt. Soc. Am. 32, 486 (1942).

${ }^{16}$ A. Lien, Liq. Cryst. 22, 171 (1997).

${ }^{17}$ V. V. Belyaev and V. G. Chigrinov, Appl. Opt. 32, 141 (1993).

${ }^{18}$ J. M. Geary, J. W. Goodby, A. R. Kmetz, and J. S. Patel, J. Appl. Phys. 62, 4100 (1987).

${ }^{19}$ D. S. Seo, S. Kobayashi, and M. Nishikawa, Appl. Phys. Lett. 61, 2392 (1991).

${ }^{20}$ S. T. Wu and C. S. Wu, J. Appl. Phys. 65, 527 (1989).

${ }^{21}$ S. Nagata, E. Takeda, Y. Nanno, T. Kawaguchi, Y. Mino, A. Otsuka, and S. Ishihara, Soc. Inform. Display Technical Digest 20, 242 (1989). 